Diabetologia (1994) 37: 32-35

\title{
Aminoguanidine inhibits the development of accelerated diabetic retinopathy in the spontaneous hypertensive rat
}

\author{
H.-P. Hammes ${ }^{1}$, M. Brownlee ${ }^{2}$, D.Edelstein ${ }^{2}$, M. Saleck ${ }^{1}$, S. Martin ${ }^{1}$ K.Federlin ${ }^{1}$ \\ ${ }^{1}$ Third Medical Department, Justus-Liebig-University of Giessen, Germany \\ ${ }^{2}$ Diabetes Research Center and Departments of Medicine and Pathology, Albert Einstein College of Medicine, \\ Bronx, New York, USA
}

Summary Arterial hypertension has been identified as a major secondary risk factor for diabetic retinopathy. However, the mechanisms by which hypertension worsens retinopathy are unknown. Inhibition of advanced glycation product formation prevents the development of experimental diabetic retinopathy in normotensive diabetic rats. In this study the effect of hypertension on the rate of diabetic retinopathy development and the formation of arteriolar thrombosis was evaluated. We also evaluated the effect of aminoguanidine, an inhibitor of advanced glycation end product formation on retinal pathology of diabetic hypertensive rats. After 26 weeks of diabetes, hypertension accelerated the development of retinopathy despite a lower mean blood glucose level than in the non-hypertensive group (diabetic spontaneous hypertensive rats (SHR) $16.00 \pm 6.83 \mathrm{mmol} / \mathrm{l}$; diabetic normotensive Wistar Kyoto rats (WKY) $34.9 \pm 3.64 \mathrm{mmol} / \mathrm{l} ; p<0.0001$ ). Diabetic SHR had nearly twice as many acellular capil- laries as diabetic WKY (SHR diabetic: $91.9 \pm 7.5$ acellular capillaries per $\mathrm{mm}^{2}$ of retinal area vs WKY diabetic: $53.7 \pm 8.5$ acellular capillaries per $\mathrm{mm}^{2}$ of retinal area), and a 3.8-fold increase in the number of arteriolar microthromboses (SHR diabetic 23504 \pm $5523 \mu \mathrm{m}^{2}$ vs SHR non-diabetic $6228 \pm 2707 \mu \mathrm{m}^{2}$ ). Aminoguanidine treatment of SHR diabetic rats reduced the number of acellular capillaries by $50 \%$, and completely prevented both arteriolar deposition of PASpositive material and abnormal microthrombus formation. These data suggest that hypertension-induced deposition of glycated proteins in the retinal vasculature plays a central role in the acceleration of diabetic retinopathy by hypertension. [Diabetologia (1994) 37: $32-35$ ]

Key words Diabetic retinopathy, rat model, hypertension, SHR, aminoguanidine, glycation.
Over the past few years, hypertension has been identified as a major secondary risk factor for diabetic microvascular complications [1-3]. Hypertension accelerates the progression of diabetic nephropathy, and antihypertensive treatment has been shown to reduce declining renal function even in normotensive diabetic subjects [4].

Systolic hypertension is also a risk factor for diabetic retinopathy [5-7] and in patients with unilateral carotid

Received: 21 April 1993

and in revised form: 19 July 1993

Corresponding author: Dr. H.-P. Hammes, Third Medical Department, Justus-Liebig-University, Rodthohl 6, D-35385 Giessen, Germany artery stenosis, retinopathy is less pronounced in the affected eye [8].

The mechanisms by which hypertension worsens diabetic retinopathy are unknown. In non-hypertensive diabetic animals, inhibition of advanced glycation product formation (AGE) by aminoguanidine prevents the development of experimental diabetic retinopathy [9]. Since AGEs induce a procoagulatory state in endothelial cells in vitro [10], we hypothesized that increased deposition of glycated plasma proteins in the microvasculature of hypertensive diabetic animals would accelerate diabetic retinopathy by inducing microvascular thrombus formation.

In order to test this hypothesis, we examined the effect of aminoguanidine treatment on the retinal pathology of diabetic spontaneously hypertensive rats. 
Table 1. Physical, metabolic and retinal morphometric parameters of non-diabetic and diabetic normotensive (WKY), spontaneously hypertensive rats (SHR) and aminoguanidine-treated diabetic SHR

\begin{tabular}{|c|c|c|c|c|c|}
\hline Groups & WKYN & WKY D & SHR N & SHR D & SHR D-AG \\
\hline Body weight (g) & $459 \pm 28$ & $327 \pm 54$ & $440 \pm 32$ & $279 \pm 79$ & $241 \pm 68$ \\
\hline BP systolic (mm Hg) & $119 \pm 12$ & $135 \pm 13$ & $171 \pm 21$ & $179 \pm 17$ & $157 \pm 17$ \\
\hline Vascular PAS-pos. deposits & $0-+$ & +-++ & $0-+$ & ++-++++ & $0-++$ \\
\hline $\begin{array}{l}\text { Microaneurysms (no of retinae } \\
\text { affected) }\end{array}$ & 0 & 2 & 0 & 5 & 0 \\
\hline
\end{tabular}

ND, Not detectable; WKY N, non-diabetic Wistar-Kyoto rats; WKY D, diabetic Wistar-Kyoto rats; SHR N, non-diabetic spontaneous hypertensive rats; SHR D, diabetic spontaneous hyper-

\section{Materials and methods}

\section{Animals}

Male 6-week-old spontaneous hypertensive rats (SHR) weighing 75-90 g and their normotensive counterparts (Wistar Kyoto rats, WKY, Charles River, Wilmington, Mass., USA), were rendered diabetic by i.v. injection of $60 \mathrm{mg} / \mathrm{kg}$ body weight streptozotocin (Sigma, St. Louis, Mo., USA) after an overnight fast. SHR with blood glucose levels greater than $15 \mathrm{mmol} / \mathrm{l}$ at 1 week after injection were randomized to receive either no treatment, or $50 \mathrm{mg} / \mathrm{kg}$ body weight aminoguanidine $\mathrm{HCl}$ (Aldrich, Rochester, NY., USA) i. p. once daily.

Systolic blood pressure was $170-180 \mathrm{~mm} \mathrm{Hg}$ in SHR, measured by indirect tailcuff plethysmography in a Gould apparatus (measurements 3-5 times within 1 day). Blood pressure was also measured after 12 weeks and at the end of the study, i.e. after 26 weeks.

Rats were fed a normal diet and water ad libitum. Weekly measurements of body weight and monthly blood glucose levels after an overnight fast were determined in triplicate (Beckman Glucose Analyser, Brea, Calif., USA). A detailed description of the study design has been given previously [11].

\section{Retinal preparations}

At the end of the study, the eyes of the animals of each study group (non-diabetic Wistar Kyoto rats (WKYN): $n=7$; diabetic Wistar Kyoto rats (WKY D): $n=7$; non-diabetic spontaneous hypertensive rats (SHR N): $n=10$; diabetic spontaneous hypertensive rats (SHR D): $n=7$; diabetic spontaneous hypertensive rats, treated with aminoguanidine (SHR D-AG): $n=5$ ) were removed under deep anaesthesia and immediately fixed in $4 \%$ buffered formalin.

After retinal isolation, the samples were washed in distilled water for $75 \mathrm{~min}$. A combined digestion $-5 \%$ pepsin in $0.2 \%$ hydrochloric acid for $1 \mathrm{~h}$, then $3 \%$ trypsin in $0.2 \mathrm{~mol} / \mathrm{l}$ Tris for $3.5 \mathrm{~h}$ tensive rats; SHR D-AG, aminoguanidine-treated diabetic spontaneous hypertensive rats

- was used to isolate the retinal vessel system. The preparations were placed on glass slides, washed with distilled water, air-dried and stained with periodic acid/Schiff reagent (PAS)/haematoxylin $[9,12]$. One retina per animal was used for morphometric evaluation.

Microthromboses were quantitated by interactively measuring the arteriolar areas containing thrombotic material (i.e. homogeneous, fibrinous, weakly PAS-positive material without cellular elements) using an image analysing system with a morphometric software program (CUE 2; Olympus Opticals Europe, Hamburg, Germany) and the total for each retina was expressed as $\mu \mathrm{m}^{2}$ of the vessel area.

Quantitation of AGE was performed as described previously [9] with the exception that a DMRXE microscope (Leica, Wetzlar, Germany) was used instead of an Aristoplan microscope.

Precapillary arteriolar PAS-positive deposits at locations corresponding to fields with accumulated AGEs were graded from 0 (none) to ++++ (very prominent) on the basis of staining intensity and size.

Measurements of AGEs, thromboses and PAS-positive deposits were restricted to the arteriolar sites of the retinal network because these changes did not occur in capillaries or venoles.

Acellular capillaries were quantitated by a modification of the method of Engerman and Kern [13]. Using a grid of 100 fields, 10 microscopic fields covering a total area of $6.76 \mathrm{~mm}^{2}$ of retinal area were scored for the presence of acellular occluded vessels (integration ocular Olympus/400 $\times$ magnification). Each field containing acellular capillary segments was recorded as positive, and values were normalized to $\mathrm{mm}^{2}$ of retinal area.

Retinae were scored for the presence or absence of saccular microaneurysms.

The numbers of endothelial cells and pericytes were counted in ten randomly-selected fields of each retina using the morphometry software program of the image analysing system. Cells were differentiated according to the criteria of Kuwabara and Cogan [12]. Fields covering an average of $45000 \mu \mathrm{m}^{2}$ of capillary area per retina examined were selected to contain only capillaries (no larger vessels) at a relative capillary density of $20-35 \%$ to avoid area underestimation by capillary overlap. The numbers 
of both cell types were automatically corrected to the relative capillary density by the morphometric software program (CUE 2) (number of cell per $\mathrm{mm}^{2}$ of capillary area). All morphometric evaluations were performed independently by two observers unaware of the identity of the samples being examined.

\section{Statistical analysis}

All parameters are given as mean \pm SD. The significance of differences between groups was tested using one way analysis of variance and the Student-Newman-Keuls test [14].

\section{Results}

The physical, metabolic and retinal morphometric parameters of the rats studied are given in Table 1 . After 26 weeks of diabetes, treated (SHR D-AG) and untreated diabetic (SHR D) animals had similar glucose levels (SHR D $16.0 \pm 6.8 \mathrm{mmol} / 1$ vs SHR D-AG $19.0 \pm 10.9 \mathrm{mmol} / \mathrm{l} ; p \mathrm{NS})$. Mean blood glucose in WKY D was significantly higher $(34.9 \pm 3.6 \mathrm{mmol} / \mathrm{l}$; $p<0.0001$ vs SHR D).

Aminoguanidine treatment had no effect on the weight gain of the diabetic groups (SHR D $279 \pm 79 \mathrm{~g}$ vs SHR D-AG $241 \pm 68 \mathrm{~g} ; p$ NS).

No difference in systolic blood pressure was found (SHR D $179 \pm 21 \mathrm{~mm} \mathrm{Hg}$ vs SHR D-AG $157 \pm 17 \mathrm{~mm}$ $\mathrm{Hg} ; p \mathrm{NS})$.

Multiple microthromboses in small, precapillary arterioles were observed in diabetic SHR, covering a total of $23504 \pm 5223 \mu \mathrm{m}^{2}$ of arteriolar area, in contrast to non-diabetic SHR $\left(6228 \pm 2707 \mu \mathrm{m}^{2} ; p<0.001\right)$ and normotensive WKY diabetic rats, where microvascular thromboses were only found occasionally. Aminoguanidine treatment completely normalized microthrombus formation (SHR D-AG $4689 \pm 4102 \mu \mathrm{m}^{2} ; p$ NS vs SHR N).

PAS-positive deposits were prominent $(++-$ ++++ ) in diabetic SHR, and $0-+$ in SHR N.Precapillary arteriolar autofluorescence was $59.5 \pm 4.2$ arbitrary units (AU) in SHR N and $153 \pm 19.5$ AU in SHR D $(p<0.0001)$. Aminoguanidine treatment markedly reduced these deposits in corresponding regions of precapillary arterioles $(0-++)$.

Retinae from diabetic SHR showed an almost 20fold increase in acellular, occluded capillaries compared to non-diabetic SHR (SHR D $91.9 \pm 7.5$ vs SHR $\mathrm{N} 4.5 \pm 0.6 ; p<0.0001)$. Although the small number of acellular capillaries found in SHR $\mathrm{N}$ was less than the small number of acellular capillaries observed in WKY $\mathrm{N}$, diabetes increased the number of acellular capillaries in hypertensive animals to a level that was nearly two times greater than in WKY. Aminoguanidine treatment in SHR reduced capillary closure by $50 \%$ $(49.3 \pm 14.5 ; p<0.001$ vs SHR D).

Diabetes-typical microaneurysmic capillary lesions (Fig.1) were observed in five of seven untreated

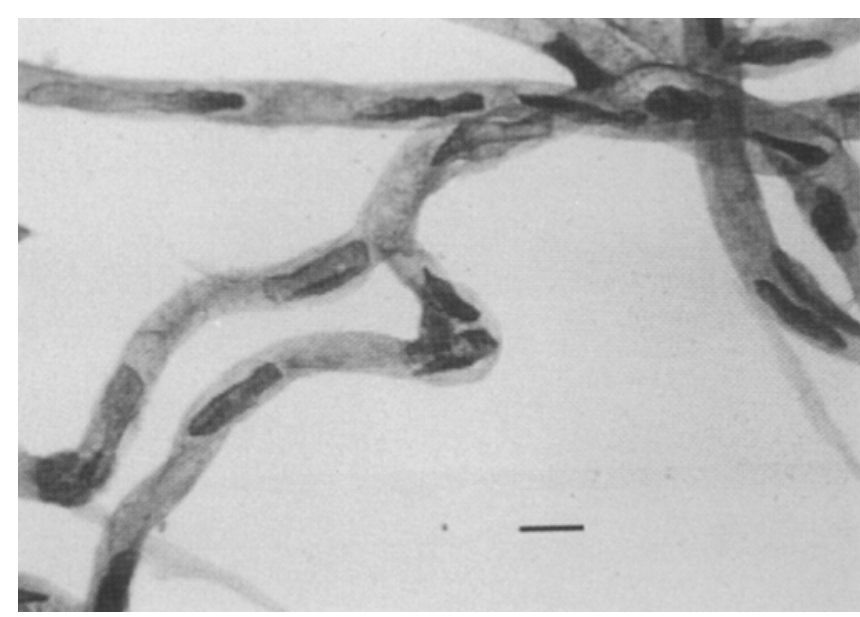

Fig.1. Pepsin/trypsin digest of the retinal vasculature prepared from an untreated diabetic SHR showing a capillary microaneurysmatic lesion. Periodic acid-Schiff and haematoxylin stain (bar $=10 \mu \mathrm{m}$ )

diabetic SHR. No such changes were present in nondiabetic or in aminoguanidine-treated diabetic SHR.

Quantitatively, hyperglycaemia induced a $27 \%$ increase in endothelial cell numbers (SHR D $3920 \pm 93$ vs SHR N $3090 \pm 124 ; p<0.0001)$ after 26 weeks and a $26 \%$ decrease in pericyte numbers (SHR D $1786 \pm 35$ vs SHR N 2255 $\pm 86 ; p=0.0003$ ). Aminoguanidinetreated diabetic SHR (D-AG) showed a significant reduction in endothelial proliferation compared to SHR $\mathrm{D}$ (SHR D-AG $2770 \pm 74 ; p<0.0001$ ). Diabetes-associated pericyte loss also appeared to be less but was not significant at a level of 0.05 (SHR D-AG $1860 \pm 41$; $p$ NS).

\section{Discussion}

This study provides evidence that coexisting hyperglycaemia and hypertension result in an acceleration of diabetic retinopathy when compared to non-hypertensive diabetic rats. Retinal changes observed in the diabetic SHR model are congruent with previous findings of Robison et al. [15] concerning vascular PASpositive deposits, pericyte loss and endothelial proliferation.

The abundance of microthromboses in diabetic SHR suggests that hypertension significantly aggravates the procoagulatory vascular state that exists in diabetes as a result of endothelial cell surface changes induced by AGE-protein accumulation [16].

Hypertension-induced increases in deposition of AGE proteins, reflected by the prominent PAS-positive deposits in the vasculature of diabetic SHR, may play a central role in this process. Recent evidence supporting this hypothesis has been provided by Panagiotopoulos et al. [17] using streptozotocin-diabetic SHR and their non-diabetic and non-hypertensive diabetic 
counterparts. They showed that the combination of diabetes and hypertension increases retinal vascular permeability by more than $80 \%$, while diabetes or hypertension alone do so by only $26 \%$ and $34 \%$, respectively.

Increased vascular plasma protein deposition and vascular occlusion such as that observed in the SHR model produce retinal neuroinfarcts that occur both in hypertensive and in diabetic primates and humans $[18$, 19].

Vascular AGE accumulation, confirmed as autofluorescence by measurement in the vasculature of SHR D, has been shown in vitro to promote increased vascular thrombogeneity via mechanisms including (i) a rapid suppression of thrombomodulin activity, which normally promotes the anticoagulatory activity of the protein $C$ pathway and (ii) increased endothelial production of a tissue-factor like procoagulant $[10,20]$.

Inhibition of $\mathrm{AGE}$ formation by aminoguanidine prevented both accelerated diabetic retinopathy and thrombus formation without affecting hypertension.

The data presented in this report demonstrate that in rats as in humans, hypertension accelerates diabetic retinopathy. Further, hypertension is required to produce arteriolar thrombosis in diabetic animals. Both of these effects, which may be causally related, are prevented by the AGE inhibitor aminoguanidine. This result suggests that hypertension-induced deposition of AGE-proteins in the retinal vasculature play a central role in the acceleration of diabetic retinopathy by hypertension.

Acknowledgements. This work was supported by NIH grant number 33861-05 (MB) and by grant number Ha 1755/1-1 from the Deutsche Forschungsgemeinschaft, Bonn-Bad Godesberg $(\mathrm{HPH})$.

\section{References}

1. Krolewski A, Canessa M, Warram JH et al. (1988) Predisposition to hypertension and susceptibility to renal disease in insulin-dependent diabetes mellitus. N Engl J Med 318: 140146

2. Klein R, Klein BED, Moss SE, Davis MD, DeMets DL (1989) Is blood pressure a predictor of the incidence or progression of diabetic retinopathy ? Arch Intern Med 149: 2427-2432

3. Drury PL (1983) Diabetes and arterial hypertension. Diabetologia 24:1-9

4. Mathiesen ER, Hommel E, Giese J, Parving H-H (1991) Efficacy of Captopril in postponing nephropathy in normoten- sive insulin dependent diabetic patients with microalbuminuria. BMJ 303: 81-87

5. Teuscher A, Schnell H, Wilson PWF et al. (1988) Incidence of diabetic retinopathy and relationship to baseline plasma glucose and blood pressure. Diabetes Care 11:246-251

6. Knowler WC, Bennett PH, Ballintine EJ (1980) Increased incidence of retinopathy in diabetics with elevated blood pressure. N Engl J Med 302: 645-650

7. Pisu E, Vitelli F, Coggi G et al. (1988) Univariate and multivariate analysis of associated factors of retinopathy in 894 Italian adult diabetics. Diabete Metab 14: 700-705

8. Duane TD, Thomas B, Field RA (1969) Symp treat diabetic retinopathy. USPHS publication 1890, Arlington, VA, GPO $657-663$

9. Hammes HP, Martin S, Federlin K, Geisen K, Brownlee M (1991) Aminoguanidine treatment inhibits the development of experimental diabetic retinopathy. Proc Natl Acad Sci USA 88: $11555-11558$

10. Esposito C, Gerlach H, Brett J, Stern D, Vlassara H (1989) Endothelial receptor-mediated binding of glucose-modified albumin is associated with increased monolayer permeability and modulation of cell surface coagulant properties. J Exp Med 170: 1387-1407

11. Edelstein D, Brownlee M (1992) Aminoguanidine ameliorates albuminuria in diabetic hypertensive rats. Diabetologia 35: 96-97

12. Kuwabara T, Cogan M (1960) Studies of retinal vascular pattern. I.Normal architecture. Arch Ophthalmol 64: 904-911

13. Engerman RL, Kern TS (1987) Progression of incipient diabetic retinopathy during good glycemic control. Diabetes 36: $808-812$

14. Sachs L (1992) Angewandte Statistik. 7 th edn. Springer, Berlin Heidelberg New York London Paris Tokyo Hong Kong Barcelona Budapest

15. Robison WG Jr, McCaleb ML, Feld LG, Michaelis OE, Laver N, Mercandetti M (1991) Degenerated intramural pericytes ('ghost cells') in the retinal capillaries of diabetic rats. Curr Eye Res 10: 339-350

16. Brownlee M, Cerami A (1981) The biochemistry of diabetic complications. Annu Rev Biochem 50: 385-432

17. Panagiotopoulos S, Gin T, Joon T et al. (1992) Antihypertensive therapy ameliorates increased albumin permeation in hypertensive diabetic rats: contrasting effects in the retina and kidney. Diabetes 41 (Suppl 1): 19 (Abstract)

18. Garner A, Ashton N, Tripathi R, Kohner EM, Bulpitt CJ, Dollery CT (1975) Pathogenesis of hypertensive retinopathy. An experimental study in the monkey. Br J Ophthamol 59 (1): $3-44$

19. Hayreh SS, Servais GE, Virdi PS (1989) Retinal arteriolar changes in malignant arterial hypertension. Ophthalmol 198: 178-196

20. Bevilacqua MP, Pober JS, Majeau GR, Fiers W, Cotran RS, Giambrone SA (1986) Recombinant tumor necrosis factor induces procoagulant activity in cultured human vascular endothelium: characterization and comparison with the actions of interleukin 1. Proc Natl Acad Sci USA 83: 4533-4537 